\title{
Schistosoma mansoni infection reduces the incidence of murine cerebral malaria
}

\author{
Judith H Waknine-Grinberg ${ }^{1,2}$, Daniel Gold ${ }^{3}$, Ariel Ohayon ${ }^{4}$, Eliezer Flescher ${ }^{3}$, Alina Heyfets ${ }^{3}$, Michael J Doenhoff ${ }^{5}$,
} Gabriele Schramm ${ }^{6}$, Helmut Haas ${ }^{6}$, Jacob Golenser ${ }^{1 *}$

\begin{abstract}
Background: Plasmodium and Schistosoma are two of the most common parasites in tropical areas. Deregulation of the immune response to Plasmodium falciparum, characterized by a Th1 response, leads to cerebral malaria (CM), while a Th2 response accompanies chronic schistosomiasis.
\end{abstract}

Methods: The development of CM was examined in mice with concomitant Schistosoma mansoni and Plasmodium berghei ANKA infections. The effect of S. mansoni egg antigen injection on disease development and survival was also determined. Cytokine serum levels were estimated using ELISA. Statistical analysis was performed using t-test.

Results: The results demonstrate that concomitant S. mansoni and P. berghei ANKA infection leads to a reduction in CM. This effect is dependent on infection schedule and infecting cercariae number, and is correlated with a Th2 response. Schistosomal egg antigen injection delays the death of Plasmodium-infected mice, indicating immune involvement.

Conclusions: This research supports previous claims of a protective effect of helminth infection on CM development. The presence of multiple parasitic infections in patients from endemic areas should therefore be carefully noted in clinical trials, and in the development of standard treatment protocols for malaria. Defined helminth antigens may be considered for alleviation of immunopathological symptoms.

\section{Background}

Malaria, an infectious disease caused by the Plasmodium parasite, is a source of enormous morbidity and mortality. Cerebral malaria (CM), seen in about 7\% of Plasmodium falciparum malaria cases, is characterized by the presence of neurological features, especially impaired consciousness [1]. The simplified explanation for CM pathogenesis is adherence and sequestration of parasitized erythrocytes, immune cells and platelets to vascular endothelial cells lining the small blood vessels of the brain. Thus, parasite-triggered cerebral inflammation is a possible cause of death from CM [2]. The immune response is critical in determining the outcome of infection [2]. CM is characterized by a Th1 response, with overproduction of some cytokines (e.g. interferon- $\gamma$, IFN $\gamma$ ), combined with underproduction of others (e.g. interleukin-10, IL-10) [3].

\footnotetext{
* Correspondence: golenser@md.huji.ac.il

'Department of Microbiology and Molecular Genetics, The Hebrew University of Jerusalem, Jerusalem, Israel, 91120
}

By analogy with the inhibition of autoimmune disease development by helminthic infections [4], it has been demonstrated, both in murine studies and in humans, that concomitant helminth infection may change the course of Plasmodium infection [5,6].

Schistosomes are parasitic trematodes found in subtropical and tropical areas. Among human parasitic diseases, schistosomiasis ranks second behind malaria in terms of socio-economic and public health importance. In many areas of the world, schistosomiasis and malaria are co-endemic: shared antigens and cross-reactive antibodies to different components of the two parasites have been detected [7]. Chronic helminthic infections are established through modulation of the host immune system. In schistosomal infection, each pair of male and female worms produces hundreds of eggs per day; eggassociated glycolipids and glycoproteins are the main target of the host humoral immune response [8]. In schistosomiasis, the early phase of infection is characterized by Th1 immune responses, which progress to a Th2 response. This pattern of cytokine expression is 
also found in non-cerebral (severe anaemic) malaria. In contrast, CM results from a predominantly Th1 response [9]. In schistosomiasis, the Th2-type responses are driven by schistosome egg antigens (SEAs) with intact carbohydrate moieties [10]. A major secretory glycoprotein, the IL-4-inducing principle from schistosome eggs IPSE (also known as alpha-1, or IPSE/alpha-1) [11], was identified as the bioactive component in Schistosoma mansoni egg extracts [12]. IPSE/alpha-1 triggers basophils to release IL-4, leading to subsequent IL-13 and additional IL-4 expression, the latter cytokine being a potential key player in Th2 biasing [8].

Several studies in murine models demonstrate a nonconsistent effect of Schistosoma infection on malaria development, which is mostly expressed as enhancement of parasitaemia [13]. Similar conclusions concerning human malaria are based on study cases in endemic areas [14]. This research examined the effect of a preexisting schistosomal infection on the development of murine CM, and the possible role of a main schistosomal antigen, IPSE/alpha-1, in the effect seen. The results demonstrate that IPSE/alpha-1 has a role in changing the course of malaria infection, leading to increased survival, and that a shift in cytokine expression is associated with $\mathrm{CM}$ reduction. Overall, this research demonstrates that CM may be alleviated by schistosomiasis.

\section{Methods}

\section{Parasites}

Schistosoma mansoni cercariae and schistosomula: an Egyptian strain of S. mansoni, kept in Puerto Rican Biomphalaria glabrata snails and ICR mice, was used throughout this work. Cercaria shedding was induced by subjecting infected, water-immersed snails to light for 1.5 hours. The cercariae were concentrated by cooling and low speed centrifugation.

The ANKA strain of Plasmodium berghei (MRA-311, CDC, Atlanta) was maintained in vivo by serial transfer of parasitized erythrocytes from infected to naive mice.

\section{Hosts}

ICR HSD (Harlan-Sprague-Dawley) male mice aged six to seven weeks were used in all experiments; eight to 10 mice per group. The mice were housed under standard light and temperature conditions and were provided with unlimited access to water and food. The experiments were carried out in accordance with institutional guidelines for animal care, by a protocol approved by the Animal Ethical Care Committee of The Hebrew University of Jerusalem, AAALAC (Association for Assessment and Accreditation of Laboratory Animal Care) accreditation number \#1285. The choice of genetically heterogenous mice was made in order to enable a more accurate reflection of CM susceptibility and the possible effect of coinfection in human beings. The validity of the CM model in outbred mice has previously been demonstrated [15]

\section{Experimental setup}

Mice were infected with 50 or 100 S. mansoni cercariae by subcutaneous injection. Four or seven weeks later, one group of each condition was infected with $5 \times 10^{4}$ $P$. berghei-parasitized red blood cells, an inoculum which leads to cerebral malaria in the majority of infected mice. Additional control groups consisted of mice infected with $P$. berghei or S. mansoni only. Worm burdens were determined by dissection of Schistosomainfected mice. Alternatively, mice were first infected with $P$. berghei and subsequently administered complete S. mansoni egg extract (SmEA) [12], egg extract from which IPSE/alpha-1 was removed (SmEA $\triangle I P S E /$ alpha1) [16] $(45 \mu \mathrm{g} / \mathrm{mouse} /$ day on days $-7,0$, and 3 postinfection; $22.5 \mu \mathrm{g} /$ mouse on day 6 post-infection), or IPSE/alpha-1 $[8,11](5 \mu \mathrm{g} / \mathrm{mouse} /$ day from day -7 to +4 post-infection), by intravenous (iv) administration. Parasitaemia was monitored every other day by thin blood smears prepared from tail blood. These were stained with a Giemsa solution and examined under a light microscope. Blood samples were taken for subsequent cytokine analysis by ELISA (Biolegend, Israel). The minimum detectable concentrations were $4 \mathrm{pg} / \mathrm{ml}, 2 \mathrm{pg} / \mathrm{ml}$, $1 \mathrm{pg} / \mathrm{ml}$, and $30 \mathrm{pg} / \mathrm{ml}$ for IFN $\gamma$, TNF, IL-4, and IL-10, respectively.

The link between immune responses, death and cerebral malaria in mouse models has previously been demonstrated [2]. Observed clinical symptoms, including an accelerated drop in temperature and death at low parasitaemia, indicate CM [17]. Parasitaemia levels, the appearance of neurological symptoms and changes in weight, temperature, and haematocrit of infected mice were evaluated. Clinical signs, which appear one to two days before death from CM, include marked coat staring, hunching, wobbly gait and reduced locomotion, convulsions, and coma. Brain pathology observed in mice dying of CM includes haemorrhages, mononuculear cell infiltrations and the development of brain oedema (Figure 1). Mice that died at a parasitaemia of up to $15 \%$ with accompanying neurological symptoms, drastic weight reduction, and a body temperature of $34^{\circ}$ $\mathrm{C}$ or below were considered to have died of CM. Mice which did not die from CM died from severe malariainduced anaemia and high parasitaemia, as has been reported in all other cases where mice do not succumb to P. berghei-induced CM [18].

\section{Histology}

Brains of non-infected and PbA-infected moribund mice were taken for histological examination. Mice were deeply anaesthetized and sacrificed by intracardial perfusion with $10 \mathrm{ml}$ ice-cold PBS. Brains were removed, fixed in 

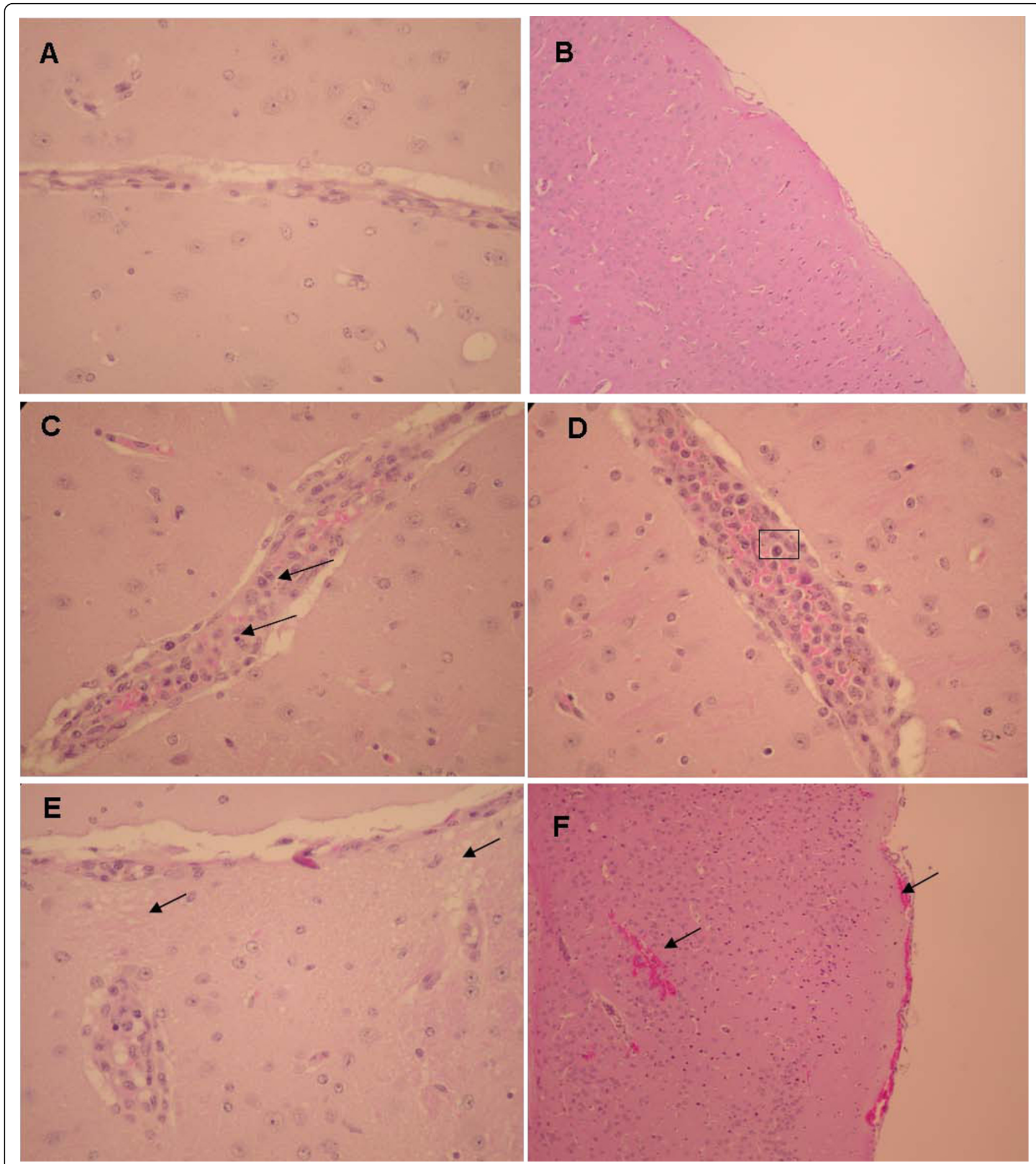

Figure 1 Brain histology of non-infected and PbA-infected ICR mice. Brains were removed from control non-infected mice $(n=10)$ or PbAinfected moribund mice $(n=10)$ on day 8 post- $\mathrm{PbA}$ inoculation. Representative sections are shown for each group. (A) Representative section of the cortex of a control mouse, demonstrating lack of vessel distention or pathology (magnification 20x). (B) Representative section of the submeningeal cortex of a control mouse, demonstrating the lack of haemorrhages or cellular infiltrations (magnification 10x). (C) Mononuclear cell aggregation in a meningeal vessel, increased cellularity, and piknotic nuclei in a prostrate mouse (magnification 20x). (D) Distended thallamic blood vessel; mononuclear cell interacting with activated endothelial cells (boxed) in a prostrate mouse (magnification 40X). (E) "Spongy" tissue - evidence of edema in a PbA-infected mouse suffering from coma (magnification 40x). (F) Meningeal haemorrahges in a PbAinfected mouse suffering from coma (magnification 10x). 

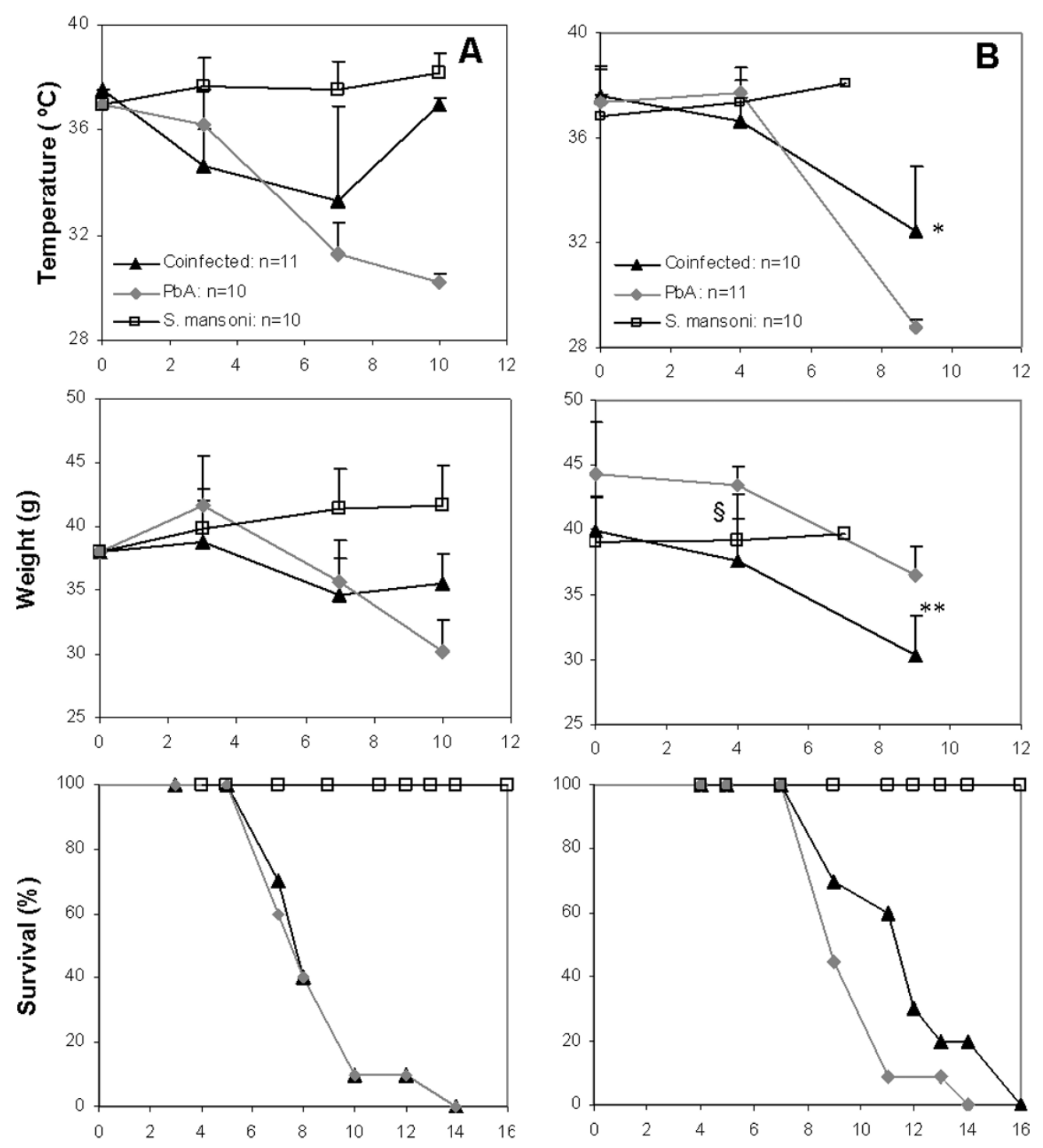

Day after $\mathrm{PbA}$ infection

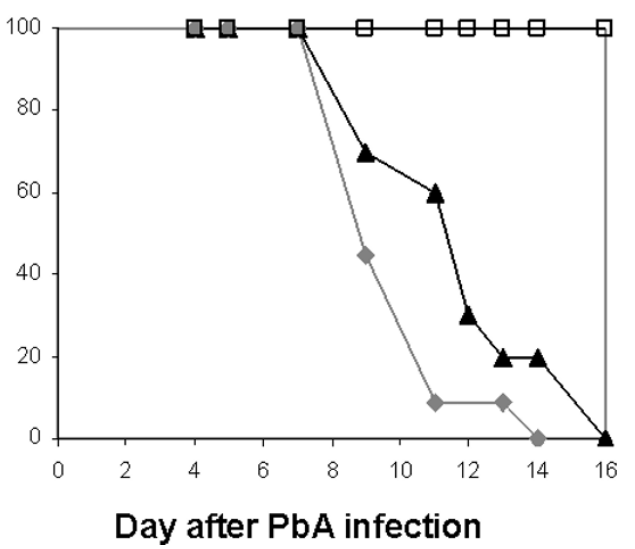

Figure 2 Changes in temperature and body weight, and survival of mice infected with PbA 4 weeks (A), or 7 weeks (B) after S. mansoni infection, and in corresponding $\mathrm{PbA}$ - and $\mathrm{S}$. mansoni infected control mice. Weight and temperature were monitored until days 9-11, when most of the deaths in the PbA-infected groups occurred. Error bars represent SD. ${ }^{*} \mathrm{p}<0.001$ (t-test), co-infected vs. S. mansoni and PbA-infected groups; ${ }^{\S} \mathrm{p}<0.0001$ (t-test), PbA- vs. co-infected mice; ${ }^{* *} \mathrm{p}<0.002$ (t-test), co-infected vs. PbA-infected mice.

a $4 \%$ formaldehyde solution, and embedded in paraffin. Paraffin-embedded tissues were cut into $5 \mu \mathrm{m}$ slices, deparaffinated, and stained with haematoxylin and eosin (H\&E) before coverslipping, according to standard procedure.

\section{Statistics}

$\mathrm{p}$ values were calculated using Students t-test.

\section{Results}

Two different infection schedules were examined, in order to determine how long after worm infection a protective effect against CM may be seen. Mice were divided into four groups, two of which were infected with $P$. berghei four or seven weeks after injection of 100 S. mansoni cercariae. The remaining two groups served as controls for S. mansoni or P. berghei infection. In mice co-infected four weeks post-S. mansoni infection, no significant effect was seen on body temperature, weight loss (Figure 2A), or CM (60\% in co-infected vs. $70 \%$ in $P$. berghei-infected mice). In contrast, when mice were co-infected seven weeks after cercariae injection, a protective effect of the pre-existing $S$. mansoni infection 
Table 1 The effect of $S$. mansoni infection on the development of cerebral malaria.

\begin{tabular}{|c|c|c|c|c|}
\hline \multirow[t]{2}{*}{50 cercariae } & \multicolumn{2}{|c|}{ P. berghei-infected mice } & \multicolumn{2}{|c|}{ Co-infected mice* } \\
\hline & Cerebral malaria & Anaemic malaria & Cerebral malaria & Anaemic malaria \\
\hline Experiment 1 & 5 & 3 & 2 & 4 \\
\hline Experiment 2 & 7 & 3 & 2 & 9 \\
\hline Total & $12 / 18$ & $6 / 18$ & $4 / 17$ & $13 / 17$ \\
\hline (\%) & $(67 \%)$ & $(33 \%)$ & $(24 \%)$ & $(76 \%)$ \\
\hline \multirow[t]{2}{*}{100 cercariae } & \multicolumn{2}{|c|}{ P. berghei-infected mice } & \multicolumn{2}{|c|}{ Co-infected mice* } \\
\hline & Cerebral malaria & Anaemic malaria & Cerebral malaria & Anaemic malaria \\
\hline Experiment 1 & 5 & 3 & 0 & 7 \\
\hline Experiment 2 & 7 & 3 & 3 & 7 \\
\hline Experiment 3 & 6 & 5 & 2 & 8 \\
\hline Total & $18 / 29$ & $11 / 29$ & $5 / 27$ & $22 / 27$ \\
\hline (\%) & $(62 \%)$ & $(38 \%)$ & (19\%) & $(81 \%)$ \\
\hline
\end{tabular}

* Mice were infected with $5 \times 10^{4}$ P. berghei-parasitized erythrocytes seven weeks after injection of 50 or $100 \mathrm{~S}$. mansoni cercariae.

was seen: co-infected mice displayed lower rates of $\mathrm{CM}$ ( $30 \%$ vs. $60 \%$ in control mice), correlated to higher body temperatures and weights, and a delay to death (Figure 2B). The haematocrit of co- and S. mansoni- infected mice was similar (an average of 53 and 54 on day 3; 47 and 49 on day 7 after $P$. berghei infection, respectively), while the haematocrit of $P$. berghei-infected mice dropped from an average 51 on day 3 to 39 on day 7 after infection. Worm loads in the S. mansoni control group were examined four or seven weeks post-infection. Worm loads were not detectable four weeks after cercariae injection. Worm loads in mice co-infected seven weeks post-S. mansoni infection were found to be slightly lower than in mice infected with $S$. mansoni only (17.6 \pm 2.3 vs. $22.6 \pm 7.3$, worms, respectively). Equal numbers of male and female worms were seen in each mouse.

The next experiment was planned to evaluate the effect of cercarial dose on the malarial infection. As shown (Figure 3), P. berghei infection led to CM in five of eight control mice $(63 \%)$; the remaining mice died of severe anaemic malaria. Two of six mice $(33 \%)$ coinfected with 50 cercariae died of CM. Infection with 100 cercariae, however, prevented cerebral malaria in all 7 co-infected mice (Table 1, Experiment 1, 100 cercariae). No significant differences in parasitaemia were seen during the first 11 days of infection, the time period in which death from $\mathrm{CM}$ occurs. On day 7 post-infection, the average haematocrit in the co-infected group was 49 \pm 9 , compared to $39 \pm 3$ in the $P$. berghei-infected group and $49 \pm 3$ in the $S$. mansoni group, indicating the absence of severe anaemia. A delay in time of death was observed in the $S$. mansoni-infected groups relative to the control group: on day 13 post-infection the majority of control mice were dead, whereas $66 \%$ of mice injected with 50 cercariae, and all of the mice injected with 100 cercariae were still alive. On day $9, P$. berghei-infected mice had lost an average $15 \pm 2 \%$ of their initial body weight, while co-infected mice lost 24 $\pm 5 \%$. This result may be attributed to the high parasite burden in the co-infected mice. In comparison, mice infected solely with $S$. mansoni gained an average $2 \pm$ $6 \%$ body weight during this period. None of the mice infected solely with $S$. mansoni died, indicating that pathology due to the helminth infection was not a cause of death in these experiments. Each experiment was repeated (Table 1). Overall, the results indicate that $S$. mansoni infection provides protection against $\mathrm{CM}$.

Serum cytokine profiles were determined at several time points in mice co-infected with $P$. berghei seven weeks after injection of $100 \mathrm{~S}$. mansoni cercariae (days 1,7 , and 10 post- $P$. berghei infection). Control groups included non-infected mice and mice infected with $S$. mansoni or P. berghei only (Figure 4). On day 1 , an increase in serum IFN $\gamma$ was seen in $P$. bergheiinfected mice $(\mathrm{p}<0.05)$, while no significant increase in IFN $\gamma$ levels was seen in co-infected mice. IFN $\gamma$ levels were below detection in non-infected and S. mansoni-infected mice. Although TNF levels rose slightly in the PbA- and co-infected groups, this change was not statistically significant. Co-infected mice displayed higher IL-4 relative to both non-infected and PbA-infected mice $(\mathrm{p}<0.05)$. IL-10 levels were similar in S. mansoni- and noninfected mice; co-infected mice displayed higher IL-10 compared to $P$. berghei-infected mice $(\mathrm{p}<0.05)$. The difference in IL-10 levels between PbA- and S. mansoniinfected mice was significant $(\mathrm{p}<0.01)$. By day 7 , IFN $\gamma$ levels had risen significantly in $P$. berghei- and coinfected mice, compared to non-infected mice $(\mathrm{p}<0.05)$ and $S$. mansoni-infected mice $(\mathrm{p}<0.01)$. Although no statistically significant differences in IFN $\gamma$ were seen when comparing $\mathrm{PbA}$ - and co-infected mice, the 


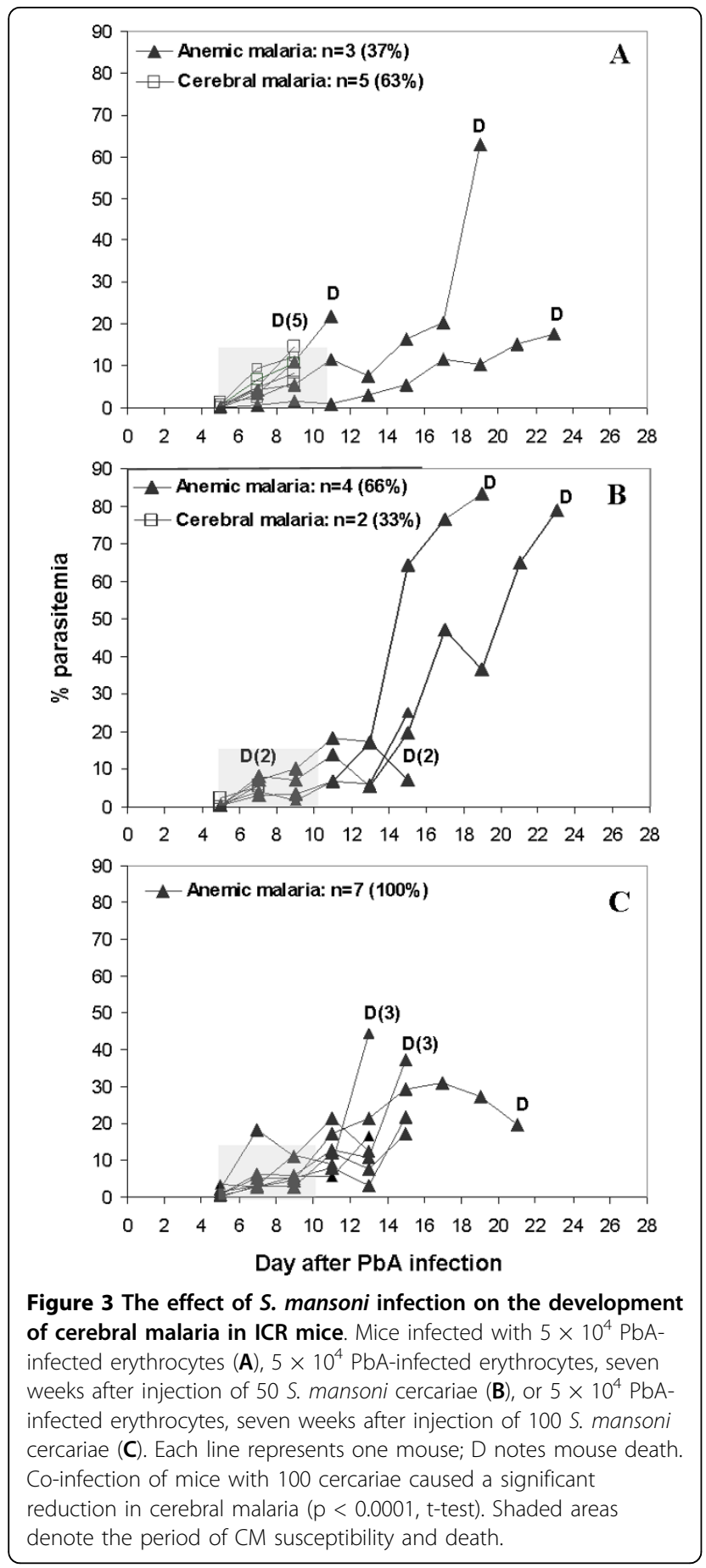

difference in TNF was significant $(\mathrm{p}<0.05)$. Co-infected mice showed a dramatic rise in IL-4 $(\mathrm{p}<0.05)$; this rise in serum IL-4 (day 7 compared to day 1, corresponding to days 42 and 36 of S. mansoni infection) is presumably due to an egg-induced Th2 response. IFN $\gamma$ was higher in $P$. berghei- and co-infected mice compared to the S. mansoni-infected group $(\mathrm{p}<0.01, \mathrm{p}<0.05$, respectively), and TNF was raised in co-infected mice compared to

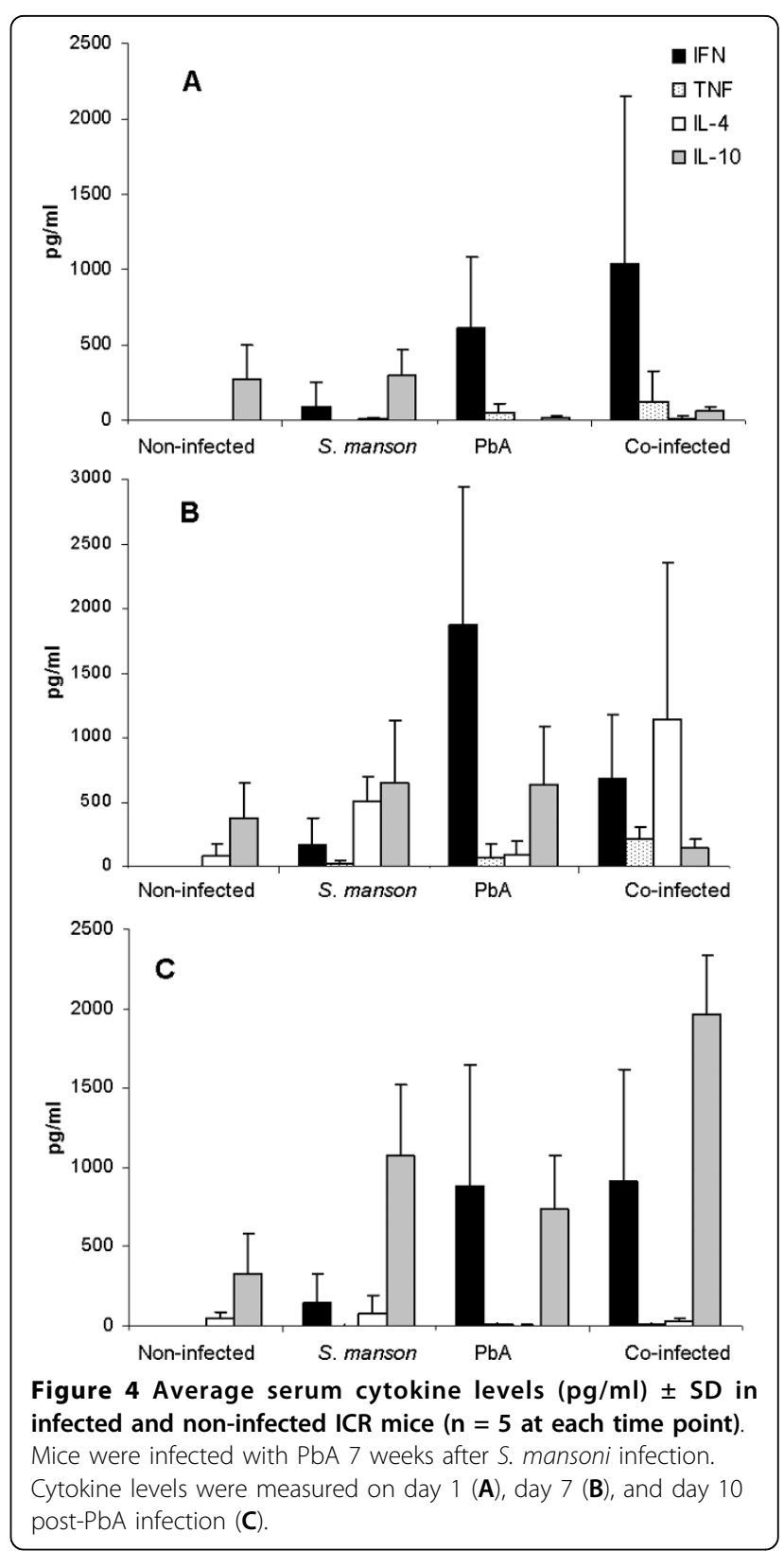

the S. mansoni-infected group ( $\mathrm{p}<0.05)$. These differences are presumably due to the ongoing inflammatory response caused by the malaria parasites. On day 10, no significant differences in IFN $\gamma$ were seen when comparing $\mathrm{PbA}$ - to $S$. mansoni-or co-infected mice, or between $S$. mansoni- and co-infected mice. Differences in IL-10 were significant $(\mathrm{p}<0.05)$ when comparing co-infected and S. mansoni- or PbA-infected mice. TNF levels were higher in co-infected vs $S$. mansoni-infected mice. No significant differences in IL-4 were seen between the groups.

The overall immune response in the different groups may be summarized using the ratio between the Th1- 


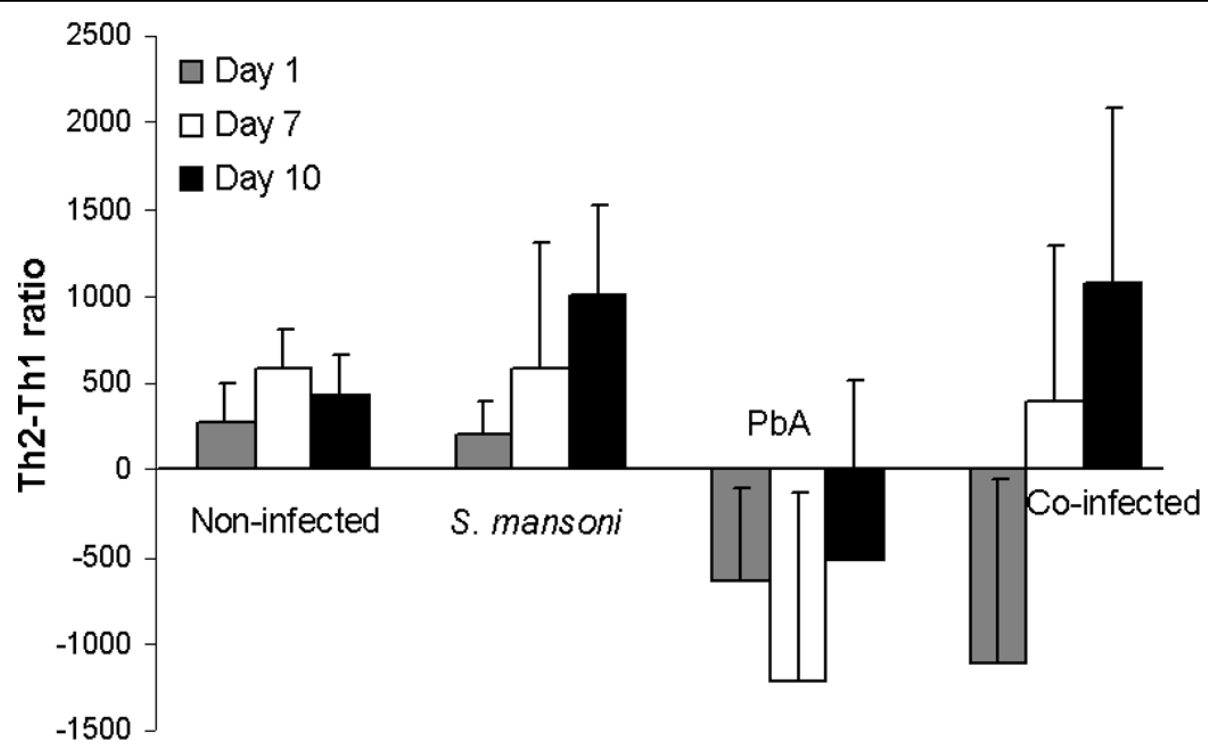

Figure 5 Average Th2-Th1 ratio. The balance between Th1 and Th2 responses was determined using the cytokine levels of each mouse, as follows: Th2/Th1 ratio $=$ (sum of IL-4 and IL-10 levels) - (sum of IFN $\gamma$ and TNF levels). Average values \pm SD are presented per day. $p<0.05$ (ttest) when comparing ${ }^{*}$ non-infected and S. mansoni-infected mice; ${ }^{*}$ non-infected and PbA-infected mice; ${ }^{\dagger}$ S. mansoni- and PbA-infected mice; ${ }^{\ddagger} S$. mansoni- and co-infected mice; ${ }^{\S} \mathrm{PbA}$ - and co-infected mice.

and Th2-group cytokines examined (Figure 5). IL-4 and IL-10 are signature Th2 cytokines; IL-4 is critically involved in biasing the immune reaction toward a Th2 phenotype. In mice which develop severe anaemic malaria, a transient early rise in IFN $\gamma$ typically occurs, while in mice which develop CM, IFN $\gamma$ levels remain high as the result of immune deregulation [2]. The results show that $P$. berghei infection caused an immediate, significant shift in the Th response of infected mice: both $P$. berghei-infected and co-infected groups showed skewing towards a Th1-type response when cytokine ratios were compared to non- or $S$. mansoni-infected mice ( $\mathrm{p}<0.01, \mathrm{p}<0.05$, respectively). However, on day 7, P. berghei-infected mice still showed a clear Th1 shift, compared to non-infected and $S$. mansoni-infected mice ( $\mathrm{p}<0.01)$; co-infected mice displayed a prevalently Th2 response $(\mathrm{p}<0.05)$. On day 10 , the difference in Th1 response in $P$. berghei- vs. S. mansoni-infected mice was still apparent $(\mathrm{p}<0.05)$, but no difference was seen when comparing $P$. berghei- and co-infected mice. Overall, the results hint at a Th2 shift caused by the preexisting schistosomal infection.

Our next step was to examine the effect of IPSE/ alpha-1 administration and injection of intact or IPSE/ alpha-1-depleted egg extract, on the course of $P$. berghei infection. Two protocols were used to determine the effect of IPSE/alpha-1 on CM. In the first experiment, 5 $\mu \mathrm{g} / \mathrm{mouse}$ IPSE/alpha-1 was injected i.v. from day -4 to day +7 post-infection. Although antigen injection did not improve the rate of $\mathrm{CM}$ (50\% of control and $75 \%$ of treated mice; Figure 6A), a significant delay in death was seen. The median survival was 15 days among treated mice, compared to 8 days in the control group (Figure $6 \mathrm{~B})$. In order to verify the role of IPSE/alpha-1 on the course of the disease, mice were injected with SmEA or SmEA $\triangle I P S E / a l p h a-1$. Both extracts were administered by i.v. injection at a dose of $45 \mu \mathrm{g} /$ mouse on days $-7,0$ and +3 followed by $22.5 \mu \mathrm{g} /$ mouse on day +6 postinfection. Administration of SmEA had a slight effect on CM development, as did SmEA $\triangle$ IPSE/alpha-1 injection (Figure 7), with the rate of CM dropping from $90 \%$ in the control group to $70 \%$ and $80 \%$, respectively. As in the previous experiments, IPSE/alpha-1 delayed mouse death. The complete extract was more effective than the extract lacking IPSE/alpha-1 (Figure 7).

\section{Discussion}

In malaria-endemic areas, infections caused by intestinal, schistosomal or filarial parasites commonly coexist with malaria in the same patient $[19,20]$. There has been much speculation as to an immunological aspect of the effect of schistosomes, which induce a host Th2 response [21], on malaria parasites.

In the present study, we investigated the effect of schistosomal parasites or their antigens on the development of the Th1-associated cerebral malaria, using the established procedure for murine CM induction. As S. mansoni affects mainly the liver, and Plasmodium infects hepatocytes prior to infection of the red blood cells, a more accurate experimental setup would have been to 

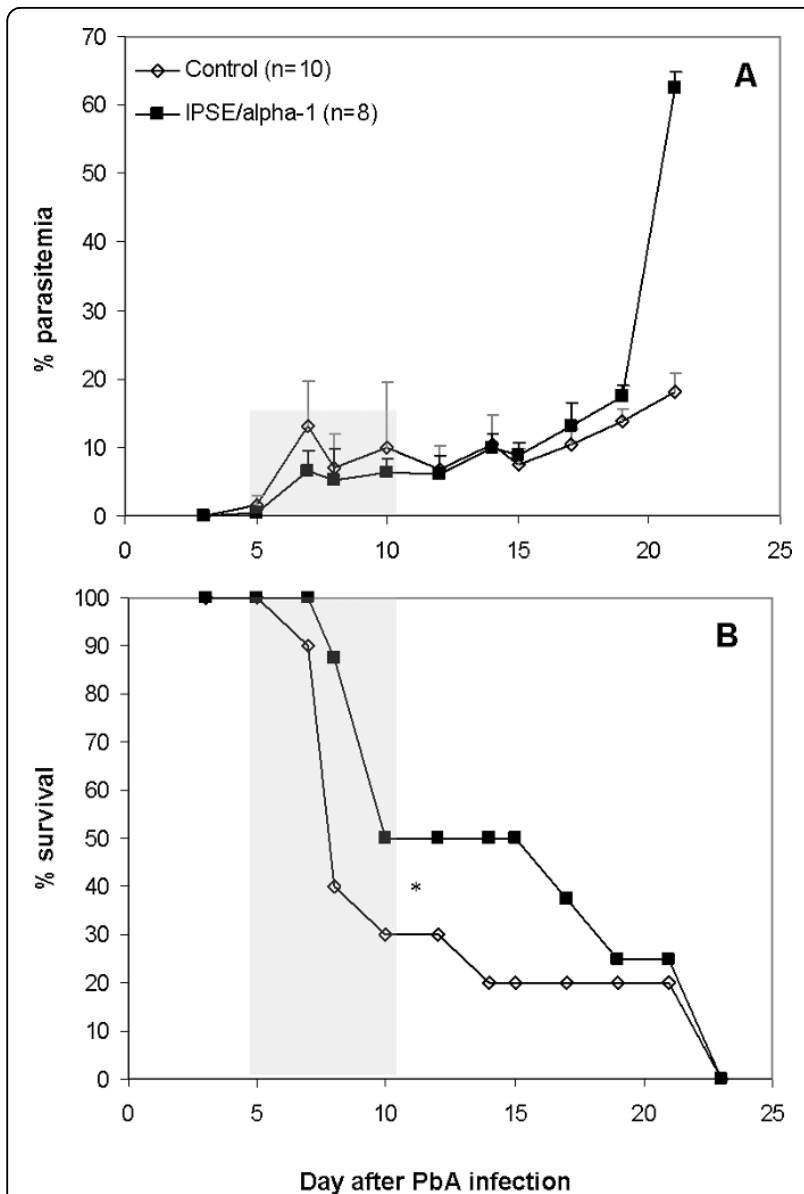

Figure 6 Parasitaemia (A) and survival (B) of mice administered IPSE/alpha- 1 by i.v. injection from day 4 before $\mathrm{PbA}$ infection to day 7 post-infection. Control mice were injected with PBS.

*Significant difference in delay to death $(p=0.005$, t-test). Shaded areas denote the period of CM susceptibility and death.

use the full plasmodial life cycle, i.e. starting from infection of the mice by the bite of infected mosquitoes. In case of a full-cycle infection, the Th2 response to schistosome eggs would likely extend to sporozoite- and liverstages of plasmodia. In analogy, it has been demonstrated that pre-treatment to tip the cytokine balance towards a Th1 response significantly reduces the malaria parasite load in the liver [22]. Thus, we suppose that the schistosomes would most likely affect the development of plasmodial sporozoites via local interactions in the liver as well as general immunomodulation.

No significant difference in $\mathrm{CM}$ rate or in survival was seen when comparing $P$. berghei-infected and coinfected mice 4 weeks post-S. mansoni infection. In contrast, concomitant infection seven weeks post-cercariae injection caused a marked reduction in CM. The lack of effect in the first case may be explained by the fact that no mature worms are seen by four weeks after cercariae injection. During the prepatent period of $S$. mansoni
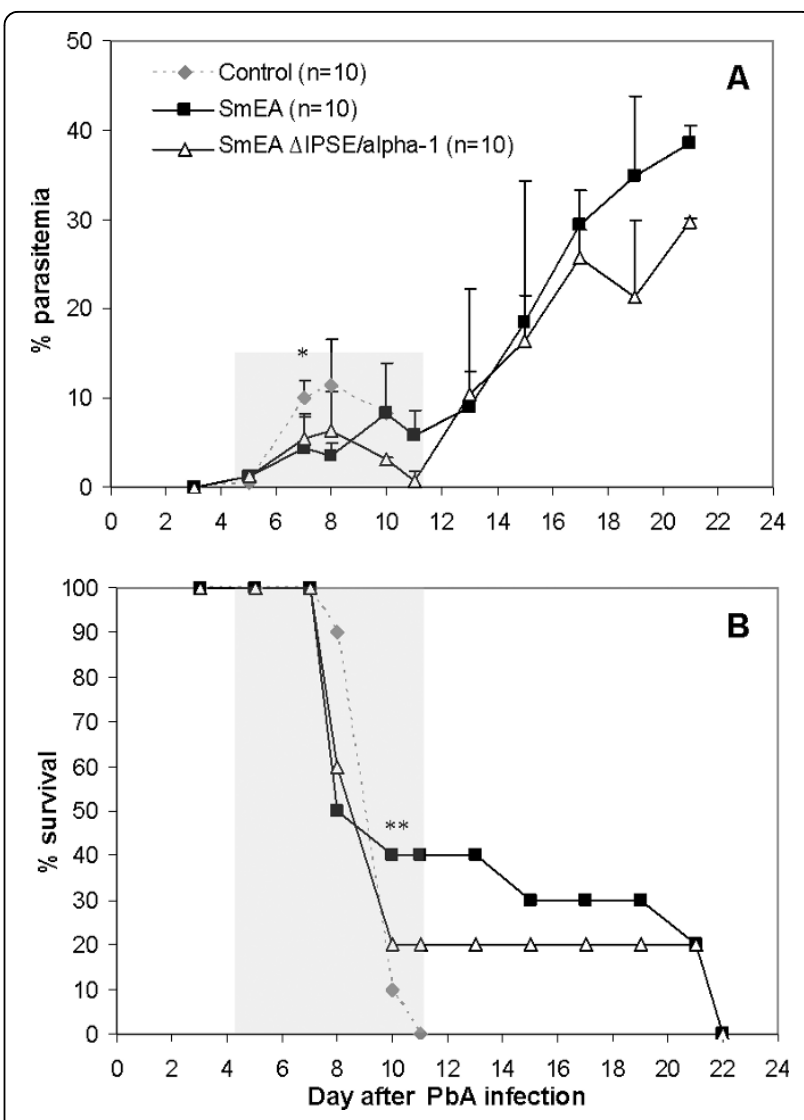

Figure 7 Parasitaemia (A) and survival (B) of control mice $(n=$ 10) and mice administered complete $S$. mansoni egg extract (SmEA; $\mathbf{n}=10$ ) or egg extract from which IPSE/alpha-1 was removed (SmEA $\triangle I P S E / a l p h a-1 ; n=10$ ), by i.v. injection, on days 7 and 0 before PbA infection and days 3 and 6 postinfection. Control mice were injected with PBS. *Parasitaemia was slightly lower in mice treated with SmEA or SmEA $\triangle I P S E / a l p h a-1$ compared to control mice, on days 7-10 post-infection $(p<0.05$, ttest). ${ }^{* *} \mathrm{~A}$ significant delay to death was seen between control and treated mice ( $p<0.05$, t-test), but not between the treated groups ( $p=0.2$, t-test). Shaded areas denote the period of CM susceptibility and death.

infection, the first 4-5 weeks following exposure to cercariae, the immune response is primarily Th1 in nature, while an egg antigen-specific Th2 response is seen by eight weeks post-infection [23]. IFN $\gamma$ production is essential for CM development [2,17]. Accordingly, each stage of schistosomal infection may affect the development of CM in a different manner: we assume that the initial Th1 response caused by the worms prevented any ameliorating effect. During the patent phase of schistosomal infection (7 weeks after injection of cercariae), a decreased rate of cerebral malaria was accompanied by higher body temperature and less severe weight reduction. Analysis of cytokine levels revealed that the preexisting $S$. mansoni infection caused a Th2 shift, which translated to the improved disease profile. This shift is 
probably the result of an overall change in the balance of cytokines, chemokines and adhesion molecules which participate in the pathogenesis of CM. Therefore, an estimate of a several factors (e.g. a sum of Th1 vs. Th2 cytokines) will better predict and correlate to the clinical situation.

Additional cytokine analysis 4 weeks after S. mansoni infection may demonstrate that the Th1 to Th2 shift has not yet occurred. However, at this stage eggs are not yet deposited. As mature egg, deposition is the major stimulus for the production of Th2 cytokines in murine S. mansoni infection [24], this is the reason for our experimental design. The effect of co-infection on the rate of $\mathrm{CM}$ was reduced when mice were injected with a lower number of cercariae (50 vs.100), presumably because of the less pronounced immune response expected to have been elicited upon helminth infection.

Egg antigens are crucial in the pathology caused by schistosomes [10,25]. In an established $S$. mansoni infection, egg-laying by the adult female worms causes a Th2 shift in the host immune response. SmEA is a complex mixture of both structural and secretory egg antigens, the latter of which are thought to react primarily with host immune cells [26]. IPSE/alpha-1, a secretory glycoprotein present only in mature eggs, is a general activator of both human and murine basophils, triggering their degranulation and IL-4 production as well as the release of IL-13 and histamine $[12,15]$. Depletion of IPSE/alpha-1 from SmEA completely abrogates the SmEA-induced basophil activation [12]. The immunogenicity of IPSE/alpha-1 is due in part to the presence of glycans that carry one or more Lewis X motifs, which, in the context of glycan conjugates, induce production of Th2-associated mediators such as IL-10 and prostaglandin E2 [8]. Egg glycolipid and glycoprotein epitopes found in both adult and larval schistosomes are the predominant targets of the humoral immune response in schistosomiasis [8]. The activity of IPSE/alpha-1 is independent of basophil preconditioning due to prior infections, and IL-4 production can be induced in basophils in the liver, a major site of $S$. mansoni egg deposition [27].

In our experiments, injection of IPSE/alpha-1 caused a consistent delay in mouse death compared to non-treated mice, and a slight reduction in parasitaemia during the first week of infection $(\mathrm{p}<0.05)$. In contrast to $S$. mansoni infection, no significant reduction in CM was induced by injection of SmEA or IPSE/alpha1. Delay in death, however, was pronounced upon injection of SmEA, an effect which was reduced upon injection of SmEA $\triangle$ IPSE/alpha-1 suggesting a role of IPSE/alpha-1. Whether the prolonged survival of the P. berghei-S. mansoni co-infected mice is due an anti-inflammatory effect of IPSE/alpha-1-induced IL-4 (and IL-13) remains to be determined. Nevertheless, this is an obvious assumption, because apart from the direct polarization of naïve $\mathrm{T}$ helper cells towards the Th2 phenotype, IL-4 has been shown to have a variety of anti-inflammatory effects, such as inhibition of the LPS-induced release of IL- 6 , TNF, and IL1- $\beta$ from monocytes [28], inhibition of Th17-development [29] and together with IL-13 the alternative activation of macrophages [30]. The observation that schistosome infection was superior to administration of SmEA or IPSE/alpha-1 in preventing CM may be explained by the continuous flow of egg antigens during infection versus bolus application in the experimental set-up and/or additional immunomodulatory factors released during active infection.

It should be emphasized that the result of infection in a malaria-endemic area has a complex pattern due to the variety of pathogens and the genetic variability of the population. Despite this complexity, various investigations have provided clues regarding the effect of concomitant helminthic infection on the course of malaria. Observations in Thailand showed that co-infection with intestinal helminths increased P. falciparum incidence two-fold but decreased CM by $64 \%$ [31]. Schistosoma haematobium infection was shown to reduce malaria severity by increasing Th2 responses in P. falciparuminfected children [32]; a recent ex-vivo study indicates that helminth infections cause an increase in IL-10 but no change in TNF [33], indicating suppression of proinflammatory responses. Age infection profiles indicate that school-age children are at the highest risk of coinfection [34]; underlying schistosomiasis was associated with protection against clinical falciparum malaria in children under the age of 9 [35]. The effects of schistosomal infection were shown to be dependant on egg load as well as age. Plasmodial levels in children with light egg loads were lower when compared to children with no schistosomal infection [35], while synergy was observed in the case of high egg loads [36]. In mice, existing $S$. mansoni infection interfered with attempts to vaccinate against BCG [37] or HIV components [38]. Intestinal helminths have also been associated with protection from immunopathology. Nacher et al [39] indicate protection from $\mathrm{CM}$ in human infection with intestinal helminths. In a murine model, CBA/J mice inoculated with Brugia pahangi developed a Th2 immune response, and, as a result, a lower rate of $\mathrm{CM}$ following $P$. berghei injection [40]. In contrast, Bejon et al [20] could not demonstrate an effect of intestinal helminths on $P$. falciparum infection. It should be noted that although these studies addressed patient exposure to both Plasmodium and helminths, additional infections, a factor shown by murine models to be possibly significant [41], was not investigated.

The effect of co-infections (schistosomes, hookworms, and plasmodia) on anaemia should also be considered in 
view of competition between haematopoietic and immunological compensations [42]. In addition, the concentration of free haem, which is also affected by the activity of these parasites, is related to the expression of haem oxygenase, which prevents CM [43]. Notably there can be significant changes in the vasculature induced by schistosome infection including the development of collateral circulation, the development of portal hypertension and hepato- and splenomegaly. All may have important consequences for blood flow and for malaria parasite distribution in concomitantly infected hosts. The impact of schistosome infection on anaemia may also be important. Red cell production dynamics and the balance between mature erythrocytes and reticulocytes may have been altered by the presence of adult erythrocyteconsuming schistosomes.

\section{Conclusions}

The results of this research demonstrate that a preexisting schistosome infection may drastically affect the outcome of Plasmodium infection. Protection from CM appears to be a function of $S$. mansoni parasite load. As mentioned, microbial and parasitic infections in malariaendemic areas are not controlled and it is likely that many persons are infected by more than one parasite species (including helminths). Presence or absence of a pre-existing infection may explain why certain individuals develop cerebral malaria while others exhibit minor symptoms only. The presence of multiple parasitic infections in patients from endemic areas should, therefore, be carefully noted in future clinical trials, and in the development of standard treatment protocols for malaria infection.

\section{Acknowledgements}

This work was supported by the Barenholz Fund, the Sir Zelman Cowen Universities Fund, the Gretel B. Bloch Trust, and by Deutsche Forschungsgemeinschaft (DFG), SFB/TR22-TP A12.

\section{Author details \\ ${ }^{1}$ Department of Microbiology and Molecular Genetics, The Hebrew University of Jerusalem, Jerusalem, Israel, 91120. '2Laboratory of Membrane and Liposome Research, Department of Biochemistry, The Hebrew University of Jerusalem, Israel. ${ }^{3}$ Department of Clinical Microbiology and Immunology, Sackler School of Medicine, Tel Aviv University, Israel. ${ }^{4}$ Shraga Segal Department of Microbiology and Immunology, Ben-Gurion University of the Negev, Beer-Sheva, Israel. ${ }^{5}$ The School of Biology, The University of Nottingham, Nottingham, UK. ${ }^{6}$ Research Center Borstel, Borstel, Germany.}

\footnotetext{
Authors' contributions

JHWG participated in the design and performance of the experiments, and in the writing of the manuscript. HH, MJD and GS participated in the design of the study, production of the schistosomal antigens and writing the manuscript. DG participated in the design, performance of the in vivo experiments using S. mansoni, and in critical reading of the manuscript. AO participated in cytokine measurements. EF and $\mathrm{AH}$ participated in experiments examining plasmodial development in mice. JG initiated and coordinated the study.

All authors have read and approved the final manuscript.
}

\section{Competing interests}

MJD is the owner of BioGlab Ltd, which sells schistosome-derived material. SmEA was supplied by BioGlab for this work on a not-for-profit basis.

The authors declare that they have no competing interests.

Received: 12 August 2009

Accepted: 5 January 2010 Published: 5 January 2010

\section{References}

1. Maitland K, Newton CR: Acidosis of severe falciparum malaria: heading for a shock?. Trends Parasitol 2005, 2:11-16.

2. Hunt NH, Grau GE: Cytokines: accelerators and brakes in the pathogenesis of cerebral malaria. Trends Immunol 2003, 249:491-499.

3. Mitchell AJ, Hansen AM, Hee L, Ball HJ, Potter SM, Walker JC, Hunt NH: Early cytokine production is associated with protection from murine cerebral malaria. Infect Immun 2005, 73:5645-5653.

4. Elliott DE, Urban JF JR, Argo CK, Weinstock JV: Does the failure to acquire helminthic parasites predispose to Crohn's disease?. FASEB 2000, 14:18481855.

5. Yan Y, Inuo G, Akao N, Tsukidate S, Fujita K: Down-regulation of murine susceptibility to cerebral malaria by inoculation with third-stage larvae of the filarial nematode Brugia pahangi. Parasitology 1997, 114:333-338.

6. Muok EM, Mwinzi PN, Black CL, Carter JM, Ng'ang'a ZW, Gicheru MM, Secor WE, Karanja DM, Colley DG: Short report: Childhood coinfections with Plasmodium falciparum and Schistosoma mansoni result in lower percentages of activated T cells and T regulatory memory cells than schistosomiasis only. Am J Trop Med Hyg 2009, 80:475-478.

7. Pierrot C, Wilson S, Lallet H, Lafitte S, Jones FM, Daher W, Capron M, Dunne DW, Khalife J: Identification of a novel antigen of Schistosoma mansoni shared with Plasmodium falciparum and evaluation of different cross-reactive antibody subclasses induced by human schistosomiasis and malaria. Infect Immun 2006, 74:3347-3354.

8. Wuhrer M, Balog Cl, Catalina MI, Jones FM, Schramm G, Haas $\mathrm{H}_{\text {, }}$ Doenhoff MJ, Dunne DW, Deelder AM, Hokke CH: IPSE/alpha-1, a major secretory glycoprotein antigen from schistosome eggs, expresses the Lewis X motif on core-difucosylated N-glycans. FEBS J 2006, 273:22762292.

9. Jankovic D, Steinfelder S, Kullberg MC, Sher A: Mechanisms underlying helminth - induced Th2 polarization: default, negative or positive pathways?. Chem Immunol Allergy 2006, 90:65-81.

10. Okano M, Satoskar AR, Nishizaki K, Harn DA Jr: Lacto-N-fucopentaose III found on Schistosoma mansoni egg antigens functions as adjuvant for proteins by inducing Th2- type response. J Immunol 2001, 167:442-450.

11. Schramm G, Gronow A, Knobloch J, Wippersteg V, Grevelding CG, Galle J, Fuller H, Stanley RG, Chiodini PL, Haas H, Doenhoff MJ: IPSE/alpha-1: a major immunogenic component secreted from Schistosoma mansoni eggs. Mol Biochem Parasitol 2006, 147:9-19.

12. Schramm G, Falcone FH, Gronow A, Haisch K, Mamat U, Doenhoff MJ, Oliveira G, Galle J, Dahinden CA, Haas H: Molecular characterization of an interleukin-4-inducing factor from Schistosoma mansoni eggs. J Biol Chem 2003, 278:18384-18392.

13. Sangweme D, Shiff C, Kumar N: Plasmodium yoelii : adverse outcome of non-lethal P. yoelii malaria during co-infection with Schistosoma mansoni in BALB/c mouse model. Exp Parasitol 2009, 122:254-259.

14. Sokhna C, Le Hesran JY, Mbaye PA, Akiana J, Camara P, Diop M, Ly A, Druilhe $P$ : Increase of malaria attacks among children presenting concomitant infection by Schistosoma mansoni in Senegal. Malar J 2004, $15: 43$

15. Martins YC, Smith MJ, Pelajo-Machado M, Werneck GL, Lenzi HL, DanielRibeiro CT, Carvalho LJ: Characterization of cerebral malaria in the outbred Swiss Webster mouse infected by Plasmodium berghei ANKA. Int J Exp Pathol 2009, 90:119-130.

16. Schramm G, Mohrs K, Wodrich M, Doenhoff MJ, Pearce EJ, Haas H, Mohrs M: Cutting Edge: IPSE/alpha-1, a glycoprotein from Schistosoma mansoni eggs, induces IgEdependent, antigen-independent IL-4 production by murine basophils in vivo. J Immunol 2007, 178:6023-6027.

17. Curfs JH, Meide van der PH, Billiau A, Meuwissen JH, Eling WM: Plasmodium berghei : recombinant interferon-gamma and the development of parasitemia and cerebral lesions in malaria-infected mice. Exp Parasitol 1993, 77:212-223. 
18. Curfs $J H$, Hermsen CC, Meuwissen $J H$, Eling WM: Immunization against cerebral pathology in Plasmodium berghei -infected mice. Parasitology 1992, 105:7-14.

19. Engwerda $C$, Belnoue $E$, Grüner $A C$, Rénia L: Experimental models of cerebral malaria. Curr Top Microbiol Immunol 2005, 297:103-143.

20. Bejon P, Mwangi TW, Lowe B, Peshu N, Hill AV, Marsh K: Helminth infection and eosinophilia and the risk of Plasmodium falciparum malaria in 1- to 6-year-old children in a malaria endemic area. PLOS Negl Trop Dis 2008, 2:e164.

21. Muturi EJ, Jacob BG, Kim CH, Mbogo CM, Novak RJ: Are coinfections of malaria and filariasis of any epidemiological significance?. Parasitol Res 2008, 102:175-181

22. Chen J, Xu W, Zhou T, Ding Y, Duan J, Huang F: Inhibitory role of toll-like receptors agonists in Plasmodium yoelii liver stage development. Parasite Immunol 2009, 31:466-473.

23. Kaplan $\mathrm{MH}$, Whitfield JR, Boros $\mathrm{DL}$, Grusby MJ: Th2 cells are required for the Schistosoma mansoni egg-induced granulomatous response. J Immunol 1998, 15:1850-1856.

24. Grzych JM, Pearce E, Cheever A, Caulada ZA, Caspar P, Heiny S, Lewis F, Sher A: Egg deposition is the major stimulus for the production of Th2 cytokines in murine schistosomiasis mansoni. J Immunol 1991, 146:13221327.

25. Grau GE, Heremans $H$, Piguet PF, Pointaire $P$, Lambert PH, Billiau A, Vassalli P: Monoclonal antibody against interferon gamma can prevent experimental cerebral malaria and its associated overproduction of tumor necrosis factor. Proc Natl Acad Sci USA 1989, 86:5572-5574.

26. Caldas IR, Campi-Azevedo AC, Oliveira LF, Silveira AM, Oliveira RC, Gazzinelli G: Human schistosomiasis mansoni: Immune responses during acute and chronic phases of the infection. Acta Trop 2008, 108:109-117.

27. Mutapi F, Ndhlovu PD, Hagan P, Woolhouse ME: Anti-schistosome antibody responses in children coinfected with malaria. Parasite Immunol 2000, 22:207-209.

28. te Velde AA, Huijbens RJ, Heije K, de Vries JE, Figdor CG: Interleukin-4 (IL-4) inhibits secretion of IL-1 beta, tumor necrosis factor alpha, and IL- 6 by human monocytes. Blood 1990, 76:1392-1397.

29. Park $H$, Li Z, Yang $X O$, Chang $S H$, Nurieva $R$, Wang $Y H$, Wang $Y$, Hood L Zhu Z, Tian Q, Dong C: A distinct lineage of CD4 T cells regulates tissue inflammation by producing interleukin 17. Nat Immunol 2005, 6:11331141.

30. Gea-Sorlí S, Closa D: In vitro, but not in vivo, reversibility of peritoneal macrophages activation during experimental acute pancreatitis. BMC Immunol 2009, 10:42.

31. Nacher M: Worms and malaria: blind men feeling the elephant? Parasitology 2008, 135:861-868.

32. Lyke KE, Dabo A, Sangare L, Arama C, Daou M, Diarra I, Plowe CV, Doumbo OK, Sztein MB: Effects of concomitant Schistosoma haematobium infection on the serum cytokine levels elicited by acute Plasmodium falciparum malaria infection in Malian children. Infect Immun 2006, 74:5718-5724.

33. Hartgers FC, Obeng BB, Kruize YC, Dijkhuis A, McCall M, Sauerwein RW, Luty AJ, Boakye DA, Yazdanbakhsh M: Responses to malarial antigens are altered in helminthinfected children. J Infect Dis 2009, 199:1528-1535.

34. Brooker S, Akhwale W, Pullan R, Estambale B, Clarke SE, Snow RW, Hotez PJ: Epidemiology of plasmodium-helminth co-infection in Africa: populations at risk, potential impact on anemia, and prospects for combining control. Am J Trop Med Hyg 2007, 77:88-98.

35. Lyke KE, Dicko A, Dabo A, Sangare L, Kone A, Coulibaly D, Guindo A, Traore K, Daou M, Diarra I, Sztein MB, Plowe CV, Doumbo OK: Association of Schistosoma haematobium infection with protection against acute Plasmodium falciparum malaria in Malian children. Am J Trop Med Hyg 2005, 73:1124-1130.

36. Sokhna C, Le Hesran JY, Mbaye PA, Akiana J, Camara P, Diop M, Ly A, Druilhe $P$ : Increase of malaria attacks among children presenting concomitant infection by Schistosoma mansoni in Senegal. Malar J 2004 3:43.

37. Elias D, Akuffo H, Pawlowski A, Haile M, Schon T, Britton S: Schistosoma mansoni infection reduces the protective efficacy of BCG vaccination against virulent Mycobacterium tuberculosis. Vaccine 2005, 3:1326-34.

38. Da'Dara AA, Lautsch N, Dudek T, Novitsky V, Lee TH, Essex M, Harn DA: Helminth infection suppresses T-cell immune response to HIV-DNAbased vaccine in mice. Vaccine 2006, 24:5211-5219.
39. Nacher M, Singhasivanon P, Traore B, Vannaphan S, Gay F, Chindanond D, Franetich JF, Mazier D, Looareesuwan S: Helminth infections are associated with protection from cerebral malaria and increased nitrogen derivatives concentrations in Thailand. Am J Trop Med Hyg 2002, 66:304309.

40. Yan Y, Inuo G, Akao N, Tsukidate S, Fujita K: Down-regulation of murine susceptibility to cerebral malaria by inoculation with third-stage larvae of the filarial nematode Brugia pahangi. Parasitology 1997, 114:333-338.

41. Christensen NO, Furu P, Kurtzhals J, Odaibo A: Heterologous synergistic interactions in concurrent experimental infection in the mouse with Schistosoma mansoni, Echinostoma revolutum, Plasmodium yoelii, Babesia microti, and Trypanosoma brucei. Parasitol Res 1988, 74:544-551.

42. Casals-Pascual C, Idro R, Gicheru N, Gwer S, Kitsao B, Gitau E, Mwakesi R, Roberts DJ, Newton CR: High levels of erythropoietin are associated with protection against neurological sequelae in African children with cerebral malaria. Proc Natl Acad Sci USA 2008, 105:2634-2639.

43. Pamplona A, Ferreira A, Balla J, Jeney V, Balla G, Epiphanio S, Chora A, Rodrigues CD, Gregoire IP, Cunha-Rodrigues M, Portugal S, Soares MP Mota MM: Heme oxygenase- 1 and carbon monoxide suppress the pathogenesis of experimental cerebral malaria. Nat Med 2007, 13:703-710.

doi:10.1186/1475-2875-9-5

Cite this article as: Waknine-Grinberg et al:: Schistosoma mansoni infection reduces the incidence of murine cerebral malaria. Malaria Journal 2010 9:5.

\section{Submit your next manuscript to BioMed Central and take full advantage of:}

- Convenient online submission

- Thorough peer review

- No space constraints or color figure charges

- Immediate publication on acceptance

- Inclusion in PubMed, CAS, Scopus and Google Scholar

- Research which is freely available for redistribution

Submit your manuscript at www.biomedcentral.com/submit
Biomed Central 